\title{
Metasurface Interlaced SR CP Patch with the Capability to Change Polarization Diversity
}

\author{
Saeid KARAMZADEH ${ }^{1}$, Vahid RAFIEI ${ }^{2}$, Hasan SAYGIN ${ }^{3}$ \\ ${ }^{1}$ Electrical and Electronics Eng. Dept, Faculty of Engineering and Natural Sciences, Bahçeşehir Univ., Istanbul, Turkey \\ ${ }^{2}$ Research and Development Dept., Microwave and Antenna Group, GraphenePi Company, Istanbul, Turkey \\ ${ }^{3}$ Application \& Research Center for Advanced Studies, Istanbul Aydin University, Istanbul, Turkey
}

karamzadeh@itu.edu.tr, v.rafiei@graphenepi.com, hasan.saygin@aydin.edu.tr

Submitted August 30, 2020 / Accepted July 3, 2021

\begin{abstract}
In this work, an affordable solution for the improved performance of circular polarization diversity array antenna by helping metasurface structure (MTS) is presented. The basic structure includes a multi-input feed network which is ended to a $2 \times 5$ sequentially rotated subarray. A layer of MTS has been used to modify basic antenna characteristics of inspiring ref. [5]. This innovation is aimed to increase the bandwidth of basic antenna from $14.7 \%(5.05-5.85 \mathrm{GHz})$ to $37.8 \% \quad(4.5-$ $6.6 \mathrm{GHz}$ ), and 3-dB AR about 4\%. Employing MTS layer leads to an increase in the gain of the antenna to $15 \mathrm{dBic}$. More details of the antenna are reported in the text.
\end{abstract}

\section{Keywords}

$\mathrm{CP}$, metasurface, polarization diversity

\section{Introduction}

Circularly polarized (CP) antennas, due to their advantages including improved immunity to multipath distortion, polarization mismatch losses, and Faraday rotation effects caused by the ionosphere, have been widely employed in many wireless systems. The CP array antennas performances generally depend on radiation elements and feed network characteristics. Many feed networks designed to improve CP array antennas have been presented [1-11]. In order to improve both impedance and axial ratio (AR) bandwidth, the sequentially rotated (SR) feed network technique has been used by many researchers. [1-6]. Disability to change polarization diversity can be introduced as a disadvantage of SR network technique. In other words, the SR feed only provides polarization diversity (LHCP or RHCP). However, in [5] and [6] antennas with capability to change polarization diversity have been reported, but they suffer problems such as a low bandwidth and gain [5] and a large profile [6]. Recently, metamaterials (MTMs) have been extremely investigated to develop the performance of the $\mathrm{CP}$ patch antenna including overall size reduction and increment in bandwidth and gain [7-11].

In [7], an antenna is reported which consists of two parts: i) A metasurface structure (MTS) superstrate consists of a $3 \times 4$ arranged array of rectangular patches printed on a dielectric substrate, and ii) a diagonal slot antenna which is etched in the ground plane. The impedance bandwidth was $33.7 \%$ from 4.2 to $5.9 \mathrm{GHz}$, and $3-\mathrm{dB}$ AR bandwidth was $16.5 \%$ from 4.9 to $5.9 \mathrm{GHz}$ with an average gain of 5.8 dBic. In [10], an MTS-based antenna composed of a square patch whose two opposed corners are chamfered and sandwiched between a $4 \times 4$ arrangement of square metal plates and the ground plane. In [11], by combining the reported element in [10] with SR technique, a broadband impedance and 3-dB AR bandwidth has been reported. Despite using MTS technique has been helped to improve characteristics of antenna, there is a vacancy for using this technique in circular polarization array antennas.

In this work, with the aim of addressing the mentioned issues such as failure to create two high-gain circular polarizations in one antenna and miniaturization of the antenna size, a three-layered array antenna is presented. The first layer includes two input ports, each of them generating an RHCP or LHCP. Each of the input ports ends to a Wilkinson power divider and is connected by metalized via holes to the top layer. The second layer includes $2 \times 5$ CP patch elements and four SR feed networks. An MTS in the third layer causes improvement of reference antenna [5] characteristics.

A comparison between the suggested antenna with reference paper [5] and also recent works is displayed in Tab. 1. As seen, the use of the MTS layer leads to increase impedance bandwidth, 3-dB AR, and gain of antenna more than $22 \%, 8.5 \%$, and $4 \mathrm{dBic}$, respectively. Despite compared [6-10], the proposed antenna has low impedance and AR bandwidth, the gain of it is much greater than theirs. Moreover, ref [6] generates only one type of CP (RHCP) and the polarization type of [10] is linear. 


\begin{tabular}{|c|c|c|c|c|}
\hline Ref. & Polarization type & BW & 3-dB Axial Ratio & Peak gain \\
\hline$[5]$ & $\begin{array}{c}\text { Circular (RHCP } \\
\text { and LHCP) }\end{array}$ & $\begin{array}{c}12.5 \%(4.95-5.61): \text { RHCP } \\
14.7 \%(5.05-5.85): \text { LHCP }\end{array}$ & $\begin{array}{c}16.5 \%(4.96-5.85): \text { RHCP } \\
19.2 \%(4.75-5.76): \text { LHCP }\end{array}$ & $\begin{array}{c}10.3 \mathrm{dBic}: \text { RHCP } \\
10.7 \mathrm{dBic}: \text { LHCP }\end{array}$ \\
\hline$[7]$ & Circular (RHCP) & $33.7 \%(4.2-5.9)$ & $16.5 \%(4.9-5.9)$ & $5.8 \mathrm{dBic}$ \\
\hline$[8]$ & Linear & $36.4 \%(4.5-6.5)$ & ---- & $14.5 \mathrm{dBi}$ \\
\hline$[9]$ & Linear & $8.3 \%(2.2-2.6)$ & ---- & $9.6 \mathrm{dBi}$ \\
\hline$[10]$ & Circular (RHCP) & $45.6 \%(4.70-7.48)$ & $41.7 \%(4.9-6.2)$ & $7.6 \mathrm{dBic}$ \\
\hline$[11]$ & Circular (RHCP) & $58.06 \%(4.4-8)$ & $21.8 \%(4.9-6.1):$ RHCP & $12.1 \mathrm{dBic}$ \\
\hline This & Circular (RHCP & $37.8 \%(4.5-6.6):$ RHCP & $23.4 \%(4.9-6.2):$ LHCP & $14.9 \mathrm{dBic}:$ :HCP \\
work & and LHCP) & $37.8 \%(4.5-6.6):$ LHCP &
\end{tabular}

Tab. 1. The comparison between proposed MTS CP antenna with recent work.

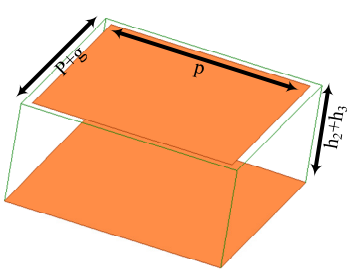

(a)

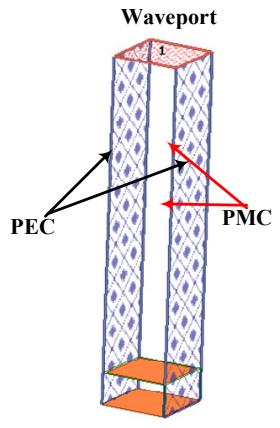

(b)
Fig. 1. The configuration of the proposed MTS and simulation boundary conditions; a) the structure, and b) simulation boundary conditions.

\section{MTS}

The configuration of the proposed MTS structure is depicted in Fig. 1. By considering the finite-sized metasurface structures as a cavity, the surface wave resonances on a finite metasurface-based antenna can be qualitatively determined by the following equation [13]:

$$
\beta_{\mathrm{SW}} \cdot L_{\mathrm{MS}}=\pi
$$

where $\beta_{\mathrm{SW}}$ represents the propagation constant of the surface wave resonances, and $L_{\mathrm{MS}}$ is the total length of the metasurface structure given by

$$
L_{\mathrm{MS}}=N \cdot P
$$

where $N$ represents the number of cells, and $P$ is the periodicity of the metasurface. Inserting equation (2) into (1), we have

$$
\beta_{\mathrm{SW}}=\frac{\pi}{N \cdot P}
$$

It was shown in reference [12] that the propagating constant of the surface waves traveling and decaying away from the metasurface is related to the decay constant $\alpha$ and the frequency $\omega$ by the following expression:

$$
\beta_{\mathrm{SW}}=\sqrt{\eta^{2} \omega^{2}+\alpha^{2}} .
$$

The propagation constant for the transverse magnetic (TM) and transverse electric (TE) waves can be expressed, respectively, as follows:

$$
\begin{aligned}
& \beta_{\mathrm{TM}}=\frac{\omega}{c} \sqrt{1-\frac{Z_{\mathrm{s}}^{2}}{\eta^{2}}} \\
& \beta_{\mathrm{TE}}=\frac{\omega}{c} \sqrt{1-\frac{\eta^{2}}{Z_{\mathrm{s}}^{2}}}
\end{aligned}
$$

where $\beta_{\mathrm{TM}}$ and $\beta_{\mathrm{TE}}$ represent the propagation constant of the TM and TE waves, respectively, $c$ is the speed of light in a vacuum, $\eta$ is the intrinsic impedance, and $Z_{\mathrm{S}}$ is the surface impedance of the metasurface structure.

As a simple unit cell model based on a simulation of the reflection phase of scattering parameters of a singleport air-filled waveguide with two perfect electrical conductor (PEC) and two perfect magnetic conductor (PMC) walls was utilized to simulate the design, as displayed in the inset of Fig. 1(b). The reflection phase of the metasurface was calculated, as illustrated in Fig. 2. The resonance frequency for the $0^{\circ}$ reflection phase was $5.4 \mathrm{GHz}$, and the $\pm 90^{\circ}$ reflection phase bandwidth was $4.75-5.75 \mathrm{GHz}$.

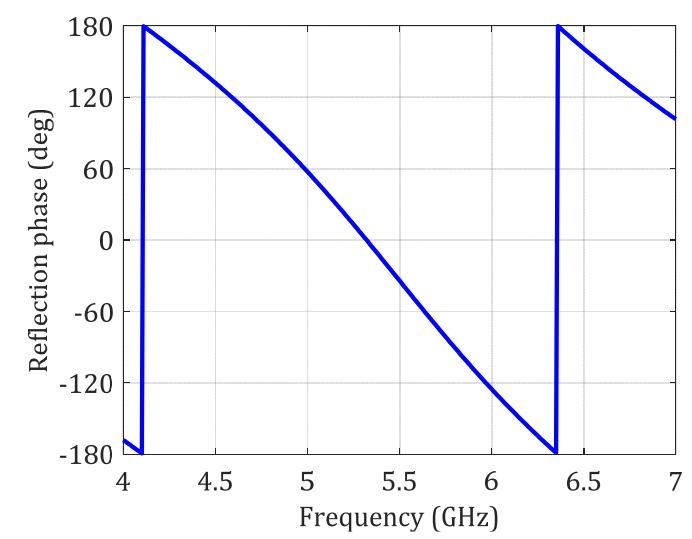

Fig. 2. Simulation reflection phase for the metasurface structure.

\section{Subarray}

The proposed CP microstrip patch subarray with SR feed network is presented in Fig. 3. It consists of $6(2 \times 3)$ square patches and two ring SR feed networks. Indeed, the structure is designed based on two ring SR feed networks with four $(2 \times 2)$ elements so that each of four elements array with its adjacent have a 90-degree rotation and adjoining elements have been overlapped together. The pro- 


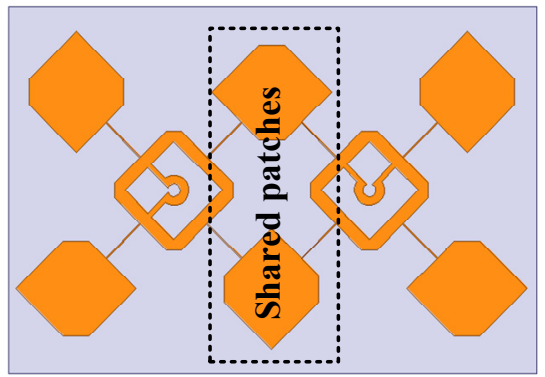

Fig. 3. The proposed CP radiation elements.

posed subarray is printed on Rogers RO4003C substrates, with $\varepsilon_{\mathrm{r}}=3.38$ and $\tan \delta=0.0027$, with a thickness of $1.524 \mathrm{~mm}$. The microstrip patches radiate $\mathrm{CP}$ with attention to feed position, and they are usually narrowband in both impedance and AR. The proposed subarray is designed relying on [5], with this difference that two ports are adjusted to generate RHCP and other two ports are considered as LHCP. For more details about $2 \times 3$ subarray refer to [5].

\section{Antenna Configuration}

The proposed CP patch array is indicated in Fig. 4. It consists of $10(2 \times 5)$ square patches with chamfering two opposite vertices [5], and four ring SR feed networks [5] which is sandwiched between ground plane and MTS. The proposed antenna is printed on three layers of Rogers RO4003C substrates with the same thickness $(1.524 \mathrm{~mm})$. The MTS is an arranged adjacent plate with periodicity of $P$ and a gap of $g$ and is designed at frequency of $5.5 \mathrm{GHz}$. The MTS plates are printed on the top side of substrate- 3 $\left(h_{3}\right)$. To obtain a low height and facilitate fabrication process, substrate- 3 is loaded above substrate- 2 without air gap. As shown in Fig. 4, to change polarization diversity in two states of LHCP and RHCP two input ports on substrate 1 are used. Each of ports ends to a Wilkinson power divider whose outputs end to two unequal length arms with 90-degree phase-difference to provide CP features.

\section{Results and Discussion}

The proposed MTS MIMO CP array antenna is capable to change polarization diversity from RHCP to LHCP by changing input ports. The proposed antenna has been fabricated at a printed circuit board (PCB) by a CNC machine with a tolerance of $0.05 \mathrm{~mm}$.

The scattering parameters of both ports were measured by Agilent Vector Network Analyzer (VNA) 8722ES. The reflection loss $\left(\mathrm{S}_{11}\right)$ of port 1 was measured while another port was loaded by a standard $50 \Omega$ and this method was used for measuring reflection loss of port 2 $\left(\mathrm{S}_{22}\right)$. The mentioned method is included in measuring $A R$, gain, and pattern of each port. In order to measure insertion loss both ports were simultaneously connected to VNA.
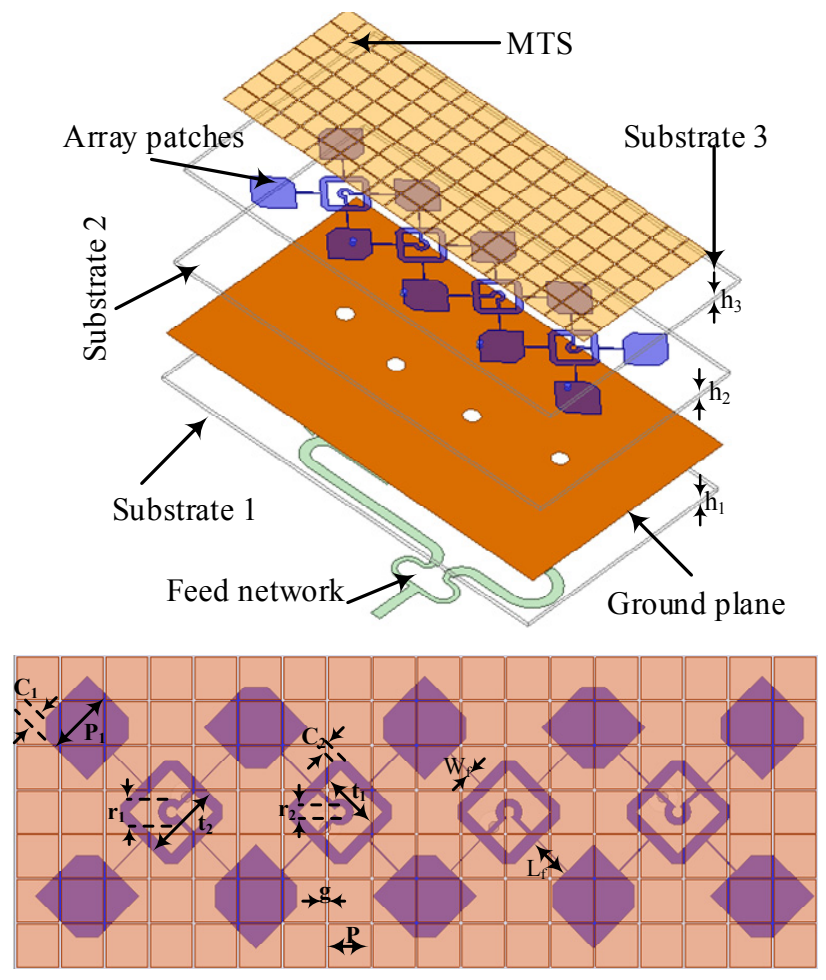

Fig. 4. Configuration of the proposed antenna $\left(h_{1}=h_{2}=h_{3}=1.524, P 1=13.8, C_{1}=3, C_{2}=1.8, t_{1}=8\right.$, $t_{2}=11, r_{1}=2.48, r_{2}=1.08, W_{\mathrm{f}}=0.2, L_{\mathrm{f}}=8, g=0.5$, $P=8$ (all values are in $\mathrm{mm})$ ).

The design procedure and results of basic antenna without MTS is utterly discussed in reference [5]. It can cover a maximum frequency range of $14.7 \%$ $(5.05-5.85 \mathrm{GHz})$ and $19.2 \%(4.75-5.76 \mathrm{GHz})$ in terms of impedance and 3-dB AR bandwidth, respectively. The basic antenna has a maximum gain of $10.7 \mathrm{dBic}$.

The comparison between simulated and measured results of scattering parameters of the proposed antenna with MTS is presented in Fig. 5(a). The proposed antenna with an isolation less than $-15 \mathrm{~dB}$ can cover an impedance bandwidth of $37.8 \%(4.5-6.6 \mathrm{GHz})$ for both ports 1 and 2 . The measured results of AR and gain of antenna for two ports are displayed in Fig. 5(b). There is an AR less than $3 \mathrm{~dB}$ in the frequency range of $21.8 \%(4.9-6.1 \mathrm{GHz})$ and $23.4 \%(4.9-6.2 \mathrm{GHz})$ for ports 1 and 2, respectively.

Therefore, by adding MTS surface waves propagating are excited to generate additional resonances and increasing impedance and AR bandwidth.

The peak gain of antenna for port 1 is $15 \mathrm{dBic}$ and for port 2 is $14.9 \mathrm{dBic}$. The measured radiation patterns of the proposed antenna at $5.5 \mathrm{GHz}$ for two ports is displayed in Fig. 6. When port 1 has been selected as an input port, the antenna radiates a half-power beamwidth (HPBW) of $25.8^{\circ}$ with an average sidelobe level (SLL) less than $16.8 \mathrm{~dB}$. These parameters for port 2 are $\mathrm{HPBW}=26.2$ and average SLL less than $16.5 \mathrm{~dB}$. The prototype of the fabricated antenna is presented in Fig. 7. 


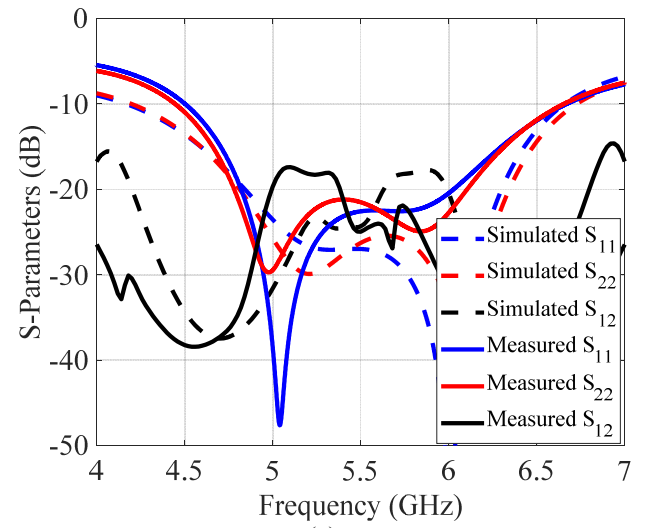

(a)

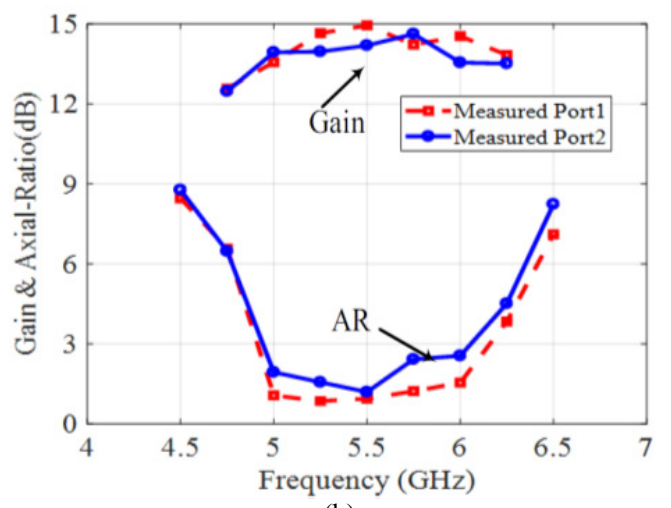

(b)

Fig. 5. Results of the proposed antenna for two ports: (a) Comparison between simulated and measured Sparameters; (b) measured gain and AR.

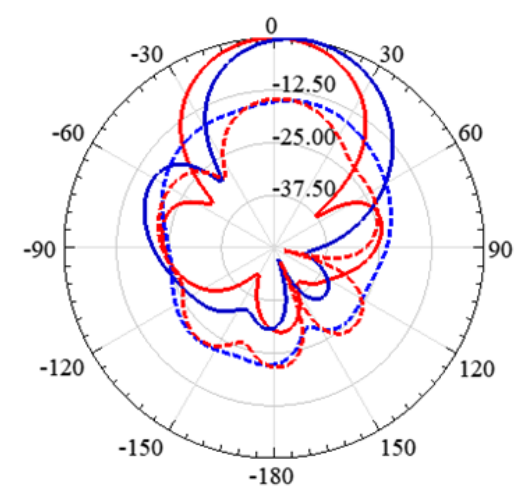

(a)

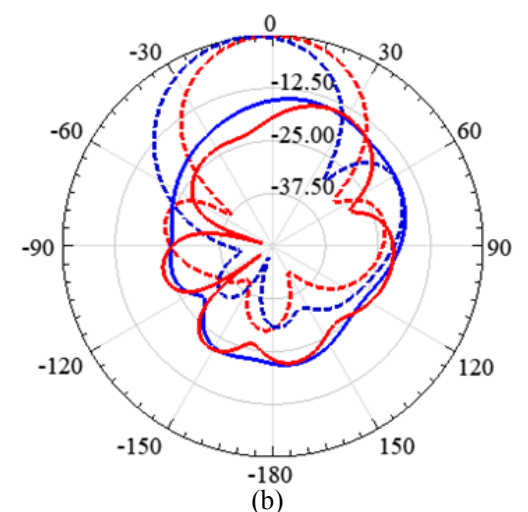

(b)

Fig. 6. The measured pattern of antenna at $5.5 \mathrm{GHz}$ (solid lines are RHCP and dashed lines LHCP, red lines are $\varphi=0^{\circ}$ and blue lines are $\left.\varphi=90^{\circ}\right)$ : a) port 1, b) port 2 .
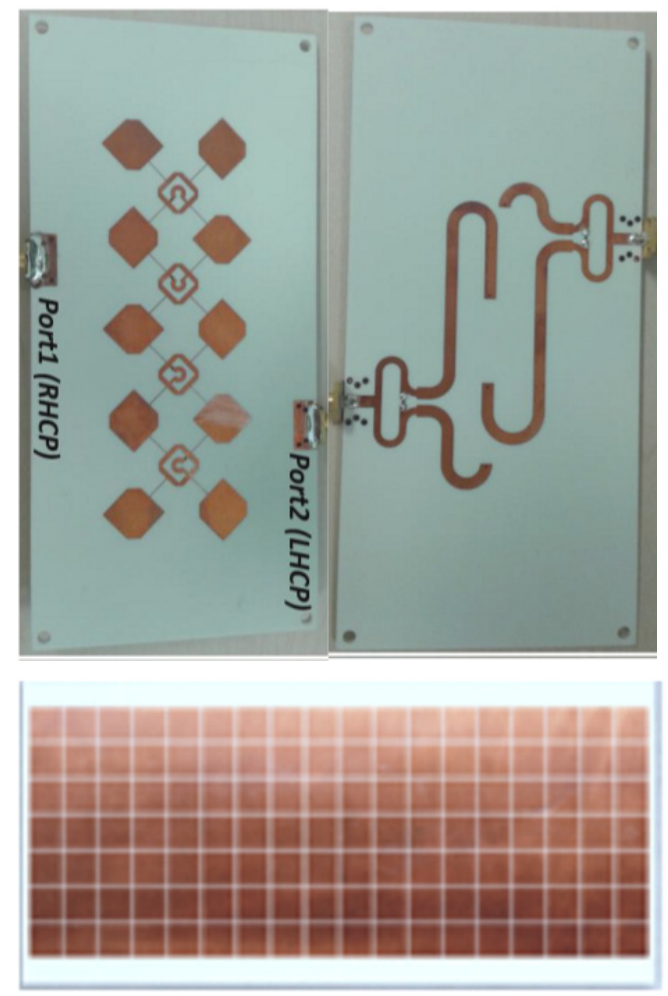

Fig. 7. The prototype of the proposed MTS antenna.

\section{Conclusion}

A miniaturized metasurface high gain microstrip patch array is understood with interlaced SR feed network. The combining patch antenna elements together lead to decrease aperture size and elements distance in antenna array. In comparison with basic work [5], incorporating MTS causes to increased BW, AR and gain of antenna more than $22 \%, 4 \%$ and $4 \mathrm{dBic}$, respectively. The most important aspect of this paper in comparison with other CP antenna, is utilizing two ports to generate two diversity of circular polarization at one antenna. Finally, combination of SR feed, interlaced patches and MTS plane causes to suppress side lobes, and also improves characteristics of antenna.

\section{References}

[1] RAFIEI, V., KARAMZADEH, S., SAYGIN, H. Millimeter-wave high-gain circularly polarized SIW end-fire bow-tie antenna by utilizing semi-planar helix unit cell. Electronics Letters, 2018, vol. 54, no. 7, p. 411-412. DOI: 10.1049/el.2018.0022

[2] KARAMZADEH, S., RAFIEI, V., SAYGIN, H. Circularly polarized aperture coupled zeroth order resonance antenna for mm-wave applications. Applied Computational Electromagnetics Society (ACES) Journal, 2017, vol. 32, no. 9, p. 789-793.

[3] BAIK, J. W., LEE, T. H., PYO, S., et al. Broadband circularly polarized crossed dipole with parasitic loop resonators and its arrays. IEEE Transactions on Antennas and Propagation, 2011, vol. 59, no. 1, p. 80-88. DOI: 10.1109/TAP.2010.2090463 
[4] DENG, C., LI, Y., ZHANG, Z., et al. A wideband sequentialphase fed circularly polarized patch array. IEEE Transactions on Antennas and Propagation, 2014, vol. 62, no. 7, p. 3890-3893. DOI: 10.1109/TAP.2014.2321380

[5] SHEN, Y., ZHOU, S. G., HUANG, G. L., et al. A compact dual circularly polarized microstrip patch array with interlaced sequentially rotated feed. IEEE Transactions on Antennas and Propagation, 2016, vol. 64, no. 11, p. 4933-4936. DOI: 10.1109/TAP.2016.2600747

[6] KARAMZADEH, S., KARTAL, M. Circularly polarized MIMO tapered slot antenna array for C-band application. Electronics Letters, 2015, vol. 51, no. 18, p. 1394-1396. DOI: 10.1049/el.2015.1784

[7] WU, Z., LI, L., LI, Y., et al. Metasurface superstrate antenna with wideband circular polarization for satellite communication application. IEEE Antennas and Wireless Propagation Letters, 2016, vol. 15, p. 374-377. DOI: 10.1109/LAWP.2015.2446505

[8] LIN, F. H., CHEN, Z. N. Low-profile wideband metasurface antennas using characteristic mode analysis. IEEE Transactions on Antennas and Propagation, 2017, vol. 65, no. 4, p. 1706-1713. DOI: 10.1109/TAP.2017.2671036

[9] LEE, Y., HA, J., CHOI, J. Design of an indoor repeater antenna with high isolation using metamaterials. Microwave and Optical Technology Letters, 2012, vol. 54, no. 3, p. 755-761. DOI: 10.1002/mop.26651

[10] TA, S. X., PARK, I. Low-profile broadband circularly polarized patch antenna using metasurface. IEEE Transactions on Antennas and Propagation, 2015, vol. 63, no. 12, p. 5929-5934. DOI: 10.1109/TAP.2015.2487993

[11] TA, S. X., PARK, I. Compact wideband circularly polarized patch antenna array using metasurface. IEEE Antennas and Wireless Propagation Letters, 2017, vol. 16, p. 1932-1936. DOI: 10.1109/LAWP.2017.2689161

[12] SIEVENPIPER, D., ZHANG, L., BROAS, R. F. J., et al. Highimpedance electromagnetic surface with a forbidden frequency band. IEEE Transactions on Microwave Theory and Technique, 1999, vol. 47, no. 11, p. 2059-2074. DOI: 10.1109/22.798001

[13] PARK, I. Application of metasurfaces in the design of performanceenhanced low-profile antennas. EPJ Applied Metamaterials, 2018, vol. 5, p. 1-13. DOI: $10.1051 /$ epjam/2018008

\section{About the Authors ...}

Saeid KARAMZADEH (corresponding author) received his M.S. and Ph.D. degree in the Department of Communication Systems, Satellite Communication \& Remote Sensing program at the Istanbul Technical University in 2013 and 2015, respectively. He won the award for the most successful Ph.D. thesis from the Istanbul Technical University. He is an Assoc. Prof. Dr at Electrical and Electronics Engineering Department, Faculty of Engineering and Natural Sciences, Bahçeşehir University currently. His research interests include remote sensing, radar, microwave, and antenna design.

Vahid RAFIEI is author and co-author of more than a dozen articles about $\mathrm{CP}$ array and beam steering antenna. He is selected as top Iranian young research in 2014-2016. Now, he is working with advance electromagnetics, microwave and antenna research group at GraphenePI company, Istanbul, Turkey. His main areas of interest in research are microstrip array antenna, beam steering and beam shaping feed networks, computational methods, microwave passive and active circuits graphene base nano structure microwave and antenna application, and RF MEMS.

Hasan SAYGIN is a professor of engineering, specializing in applied sciences, and advisor to the Chairman of the Board of trustees at the Istanbul Aydin University. He received his Ph.D. degree from École Polytechnique de Montréal, Institut de génie énergétique, Université de Montréal, and his M.Sc. degree in Nuclear Energy from the Istanbul Technical University. His working areas are nuclear energy, thermodynamic, computational fluid mechanics, energy policy, microwave, and antenna application. 